\title{
Infrared to Millimeter Spectroscopy and Imaging of Active Galactic Nuclei
}

\author{
R. Genzel, D. Lutz, E. Sturm, L. Tacconi, and N. Thatte \\ Max-Planck-Institut für Extraterrestrische Physik, Postfach 1603, \\ D-85740 Garching, Germany
}

\section{A. Sternberg}

School of Physics and Astronomy, Tel Aviv University, Tel Aviv 69978, Israel

\begin{abstract}
Observations in the $1 \mu \mathrm{m}$ to $1 \mathrm{~cm}$ wavelength band give important information on the physical processes occurring in and immediately around active galactic nuclei. Concentrating on recent results on ionic, atomic, and molecular emission lines, we discuss as examples the first $I S O$ results on the nature of ultra-luminous infrared galaxies, and near-infrared and millimeter measurements of the central 100 parsecs of the Seyfert 2 galaxy NGC 1068.
\end{abstract}

\section{Introduction}

The infrared to millimeter waveband $(1 \mu \mathrm{m}$ to $1 \mathrm{~cm})$ is a very interesting new window for the exploration of active galactic nuclei. At these wavelengths, it is possible to probe regions that are heavily obscured by line-of-sight dust. Across the waveband there are a host of spectral lines of key ions, atoms, and molecules and characteristic signatures of various types of dust particles. It is therefore possible to probe the spatial distribution, composition and dynamics of interstellar matter (ISM) in the immediate vicinity of the nucleus, in particular of the neutral ISM that cannot be observed at any other wavelength. At the shortwavelength end of the infrared, there are also stellar (absorption) features that allow observations of the (cooler) stellar component in AGNs.

Substantial improvements in sensitivity and angular resolution have occurred in the last years throughout the $1 \mu \mathrm{m}$ to $1 \mathrm{~cm}$ region. At near- and midinfrared wavelengths $(1-30 \mu \mathrm{m})$ - where observations are possible in a number of atmospheric windows from the ground - large-format, high quantum-efficiency, and low read-noise detector arrays have become available. As a result, versatile and efficient imagers, spectrometers, and imaging spectrometers in the style of optical instruments have been constructed with corresponding enormous improvements in speed and data quality. As adaptive optics and speckle imaging become easier at longer wavelengths, sub-arcsecond, near-infrared imaging has become feasible. With the launch of the Infrared Space Observatory (ISO) of the European Space Agency (ESA), a new era has begun in sensitive photometry, imaging, and spectroscopy across the entire $2.5-200 \mu \mathrm{m}$ band. Of particular 
interest for AGN work are ISO's photometric and spectroscopic capabilities. Millimeter interferometry with spatial resolutions approaching $1^{\prime \prime}$ have made it possible to map the distribution and dynamics of circumnuclear molecular gas in AGNs.

It can be extrapolated that these impressive improvements in measurement capabilities are going to continue in the next decade. In 1997, the Hubble Space Telescope will be equipped with the near-infrared camera/spectrometer NICMOS. Adaptive-optics imaging and imaging spectroscopy on the 8-m-class telescopes will explore scales around $0^{\prime \prime} .1$ or smaller. In the mid- and far-infrared, NASA's Space Infrared Telescope Facility (SIRTF) will go the next step beyond $I S O$. Interferometers will allow sub-arcsecond resolution mapping to wavelengths as long as $800 \mu \mathrm{m}$.

As examples of recent AGN science coming out in the infrared to millimeter band, we will discuss in the following first results from ISO relating to the nature of ultra-luminous IRAS galaxies, and then summarize near-infrared and millimeter imaging and spectroscopy of the central 100 parsecs of the prototypical Seyfert 2 galaxy NGC 1068.

\section{What Powers (Ultra-) Luminous Infrared Galaxies?}

One of the key discoveries of the Infrared Astronomical Satellite (IRAS) survey in the mid 1980 s was the identification of a class of very luminous $(L \approx$ $\left.10^{11.5-12.5} L_{\odot}\right)$ galaxies emitting most of their energy in the far-infrared $(30-$ $300 \mu \mathrm{m}$ ) wavelength band (for reviews see Soifer et al. 1987, Sanders \& Mirabel 1996). While these galaxies are relatively rare, their local volume density is similar to or even exceeds that of optically selected QSOs of the same luminosity (Soifer et al. 1987). Most of them appear to be interacting galaxies, or advanced mergers (Soifer et al. 1987, Sanders et al. 1988). They are very gas- and dustrich (e.g., Sanders \& Mirabel 1985, Sanders et al. 1988, Rigopoulou et al. 1996b) with typical gas masses of $\sim 10^{10} M_{\odot}$. Millimeter interferometry of some of the prototypical sources, such as Arp 220 (Wang et al. 1991), Mkn 273 (Yun \& Scoville 1995), Mkn 231 (Bryant \& Scoville 1996) and F10214+4724 (Downes et al. 1995, Scoville et al. 1995) shows that this gas is typically concentrated in the central kiloparsec or less. This translates to average hydrogen column densities exceeding $10^{24} \mathrm{~cm}^{-2}$ or visual extinctions exceeding $A_{V}=500 \mathrm{mag}$.

A key open question about these infrared-luminous galaxies is the source(s) of their luminosity. Based on their far-infrared, millimeter, and radio properties, many authors have argued that they are powered by active star formation (e.g., Rieke et al. 1985, Rowan-Robinson \& Efstathiou 1993, Condon et al. 1991). Their optical properties, on the other hand, resemble those of narrow-line active galactic nuclei (LINER or Seyfert 2), with a few broad-line examples (e.g.; Mrk 231) (Sanders et al. 1998, Veilleux et al. 1995). Some of them have compact radio nuclei (Lonsdale et al. 1993) or highly absorbed hard X-ray sources, again indicative of hidden AGNs.

With the advent of the Infrared Space Observatory (ISO), sensitive mid- and far-infrared spectroscopy has now become available as a new, very specific tool for investigating the physical processes at the nuclei of obscured galaxies. The data discussed below were taken with the short wavelength spectrometer (SWS: 
deGraauw et al. 1996) during the performance-verification (PV) phase and the first three months of the central program (CP) phase. The results are discussed in more detail in the special issue of Astronomy 8 Astrophysics dedicated to ISO (Lutz et al. 1996, Rigopoulou et al. 1996, Sturm et al. 1996, Kunze et al. 1996, Moorwood et al. 1996).

The ionic infrared emission lines toward galactic nuclei come - like optical emission lines - predominantly from photoionized gas. Hence a powerful tool for investigating whether star formation or a central AGN dominates in obscured nuclei is to study the excitation state of the mid-and far-infrared emission-line spectrum. Of particular interest are high-excitation ('coronal') lines that require a much harder radiation field than can be delivered by stars and thus are signposts for a (hidden) AGN. As a demonstration of SWS's capabilities in this regard, Fig. 1 shows two full scans of the entire $2.5-45 \mu \mathrm{m}$ spectrum of a classical starburst galaxy (M 82) and of the nearby Seyfert 2 galaxy Circinus (A1409-65). The qualitative and quantitative differences in the emission-line spectra of the two galaxies are obvious. M 82 is dominated by fairly low-ionization species ([Ne II], [S III], [S III], [Ar II]), while Circinus shows also very intense high-excitation lines ([O IV $],[\mathrm{NeV}],[\mathrm{NeVI}],[\mathrm{MgVIII}],[\mathrm{Si} \mathrm{IX}]$ ) with ionization potentials up to $320 \mathrm{eV}$ (see Moorwood et al. 1996 and Oliva et al. 1994 for more details). Figure 2 is a plot of the (dereddened) [Ne v]/[NeII] and [O IV] $/[\mathrm{NeII}]$ line ratios (or $3 \sigma$ upper limits) for 11 galaxies observed so far with SWS (Lutz et al. 1996). This includes several starburst and AGN templates, as well as three prototypical (ultra-) luminous IRAS galaxies (Arp 220, NGC 6240, NGC 3256). In all sources known to be powered by stars alone, the $[\mathrm{Ne} \mathrm{V}] /[\mathrm{Ne} I I]$ and $[\mathrm{O}$ IV $] /[\mathrm{Ne} I \mathrm{I}]$ ratios are $\leq 0.1$, while these ratios are between 0.13 and 1.5 in the two AGNs. All three luminous IRAS galaxies also have line ratios $\leq 0.1$, strongly supporting the notion that an (even moderately extinguished) AGN cannot be the main source of their luminosity. The only way out of this important constraint is to postulate that these sources contain AGNs that are hidden even at $15-30 \mu \mathrm{m}$, requiring $A_{V} \geq 100$ (i.e., $\mathrm{N}\left(\mathrm{H}_{2}\right) \geq 10^{23} \mathrm{~cm}^{-2}$ ). Against this explanation speaks the fact that such a compact ( $\leq 10 \mathrm{pc}$ scale), highly optically thick source would produce bright hot-dust emission in the $5-20 \mu \mathrm{m}$ range. All three luminous IRAS galaxies, however, emit most of their luminosity between 30 and $200 \mu \mathrm{m}$, requiring minimum (black body) source sizes of several hundreds of parsecs. It is thus very probable that Arp 220, NGC 6240, and NGC 3256 are powered mainly by stars (although one cannot exclude of course that an AGN contributes a fraction of the bolometric luminosity).

Assuming then that massive stars dominate the luminosity of Arp 220, NGC 6240 , and NGC 3256, what is the evolutionary state of the starburst? For these three and other luminous infrared galaxies, a number of near-infrared/optical studies addressing these issues can be found in the literature (e.g., Lester et al. 1988, Thronson et al. 1990, Doyon et al. 1992, van der Werf et al. 1994, Moorwood and Oliva 1994, Armus et al. 1995, 1996, Larkin et al. 1995, Genzel et al. 1995, Goldader et al. 1995, 1996). The basic common conclusion of these investigations, based on the small ratio of near-infrared recombination-line luminosity to total (far-infrared) luminosity, is that the observed far-infrared luminosity is not dominated by recently formed, massive young stars. According to these observations, if stars power these galaxies, the last period of very active star 


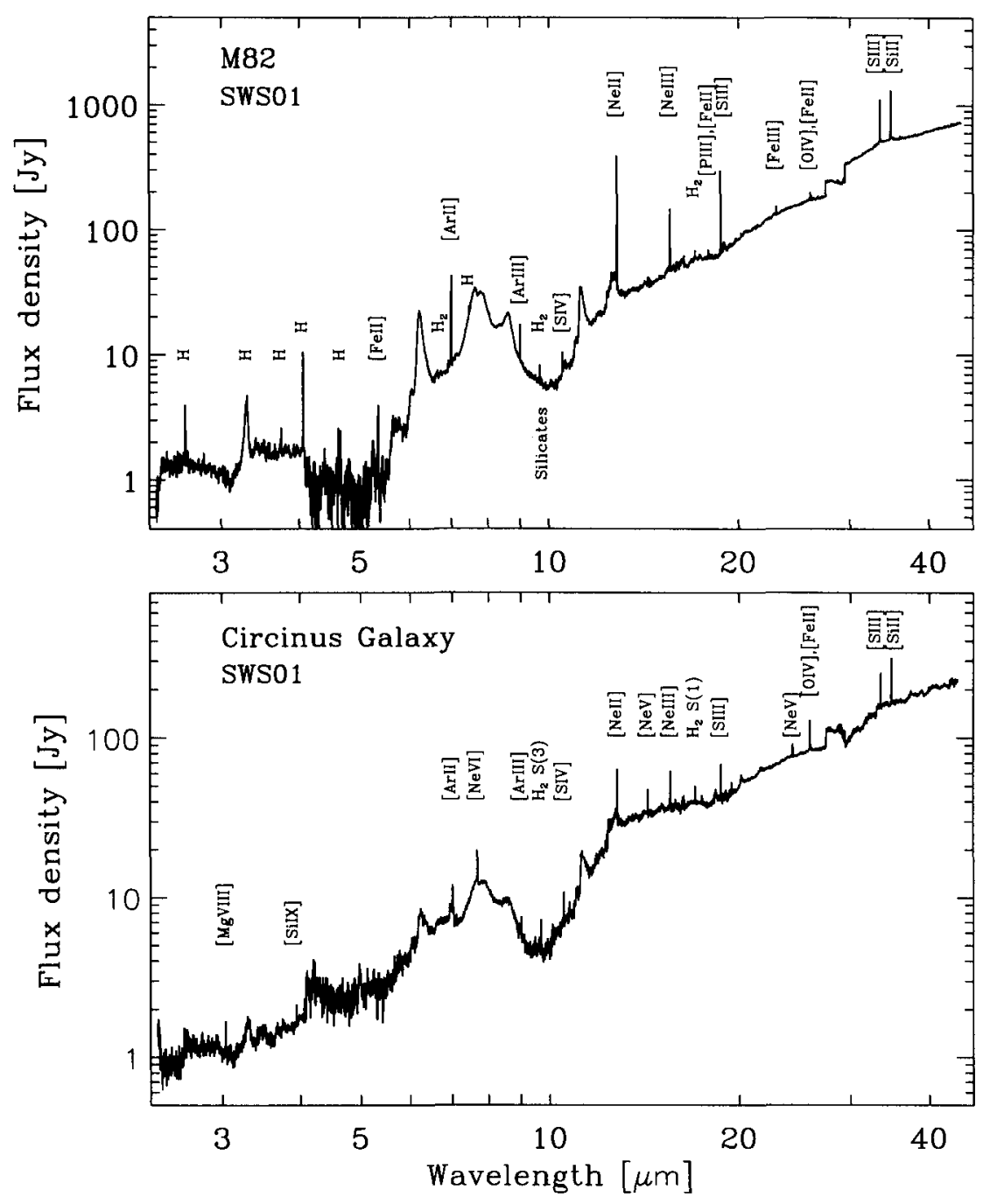

Figure 1. Top: Full 2.5-45 $\mu \mathrm{m}$ SWS spectra of the starburst galaxy M 82. Bottom: The SWS spectrum of the Seyfert 2/starburst galaxy Circinus (Moorwood et al. 1996). The jumps at $\sim 30 \mu \mathrm{m}$ are due to a change in aperture. 


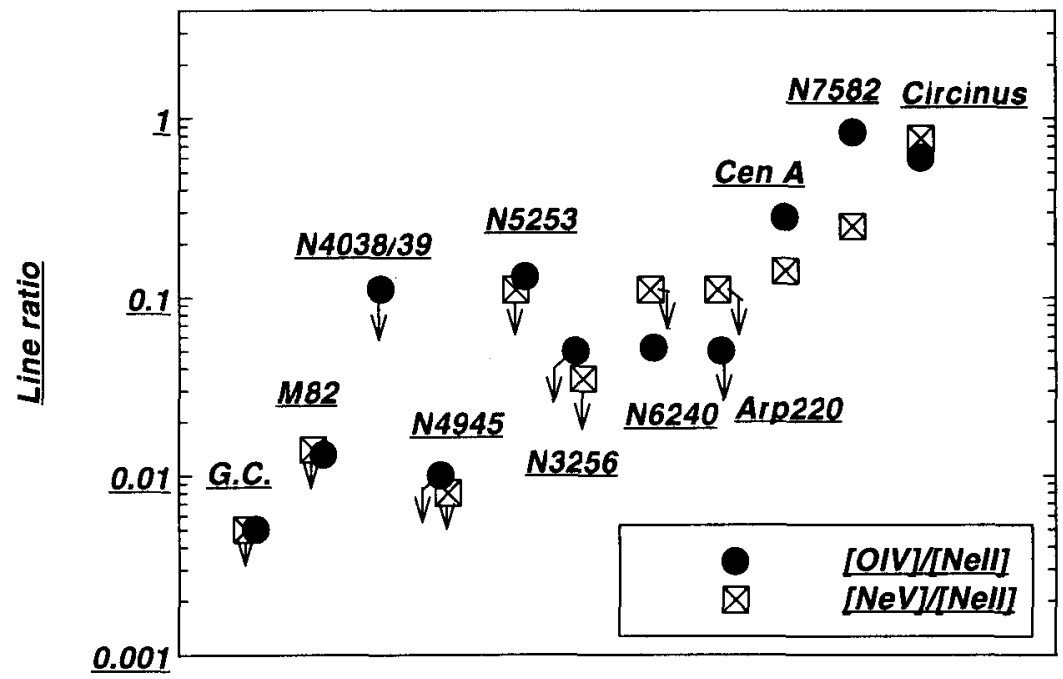

Figure 2. Dereddened flux ratios for [Ne V] $\lambda 14.3 \mu \mathrm{m} /[\mathrm{Ne} I \mathrm{I}] \lambda 12.8 \mu \mathrm{m}$ and [O IV] $\lambda 25.9 \mu \mathrm{m} /[\mathrm{NeII}] \lambda 12.8 \mu \mathrm{m}$ (or $3 \sigma$ upper limits) for 11 galaxies observed so far by ISO-SWS (Lutz et al. 1996). The excitation potentials for [Ne v] and [O IV] are $97 \mathrm{eV}$ and $55 \mathrm{eV}$, respectively. To the left are the starburst template galaxies, to the right three active galactic nuclei. The three (ultra-) luminous IRAS galaxies (NGC 3256, NGHC 6240, Arp 220) are in the middle. 
formation must have occured $\geq 10^{8}$ years ago or stars more massive than $20 M_{\odot}$ have recently not been forming.

The new ISO-SWS data now suggest that the near-infrared/optical emission is much more affected by dust obscuration than previously thought. Observations at mid- and far- infrared wavelengths (including still significant corrections for extinction with upward corrections of the observed [Ne II], [S III], etc., lines) are required to penetrate the dust enshrouding the sources of luminosity in the luminous IRAS galaxies. Lutz et al. (1996) have used the starcluster evolution code of Kovo \& Sternberg (1996) to calculate the number of stars of different type and the global $L_{B o l} / L_{L y c}, L_{K} / L_{L y c}$, and supernova rate to $L_{B o l}$ ratios as a function of time in three different star-formation histories: pure $\delta$-bursts $\left(\Delta t=10^{6}\right.$ years $)$, extended bursts $\left(\Delta t=2 \times 10^{7}\right.$ years $)$, and constant star formation. They carried out calculations for Salpeter initial mass functions $\left(d N(M) / d M \propto M^{-2.4}\right)$, solar abundances, and upper-mass cutoffs of 25,50 , and $100 M_{\odot}$. The cluster-averaged stellar spectrum was synthesized by coadding Kurucz atmospheres with the appropriate weighting of different stellar types as calculated from the Kovo and Sternberg code. The various characteristic infrared line ratios (e.g., [NeIII]/[NeII]) were then calculated with the ION photoionization code (Netzer 1993) with $\log (U)=-2.5$ and $n_{e}=300 \mathrm{~cm}^{-3}$. The results for the specific case of $m_{u}=100 M_{\odot}$ are plotted in Fig. 3, which also shows the locations of the luminous IR galaxies and starburst templates that we have observed. Constant star formation models and models with low upper-mass cutoffs $\left(m_{u} \approx 25 M_{\odot}\right)$ clearly do not fit any of the objects. On the other hand, $\delta$-bursts fit localized regions fairly well. One particularly interesting case of such a confined, local $\delta$-burst region is the overlap region in the Antennae galaxy pair (SWS: Kunze et al. 1996, LWS: Fisher et al. 1996, CAM: Vigroux et al. 1996).

The best overall fits for both starburst templates and the luminous IR galaxies observed so far are for moderately extended bursts $\left(\Delta t \approx 1-2 \times 10^{7}\right.$ years) with mean ages ranging between 1 and $7 \times 10^{7}$ years and high upper-mass cutoffs $\left(50-100 M_{\odot}\right)$. Arp 220 and NGC 6240 still require somewhat older ages than the starburst templates, but otherwise are very similar. Spatially resolved near-infrared imaging spectroscopy of M 82 (Foerster et al. 1996, Satyapal et al. 1996) and NGC 1808 (Krabbe et al. 1994, Kotilainen et al. 1996, TacconiGarman et al. 1996) shows that bursts that appear to be extended in time when their integrated emission is considered break up at high spatial resolution into a number of local, $\delta$-bursts (like giant $H$ II regions or massive star clusters) of different ages (see also Rieke et al. 1993).

There still remain substantial uncertainties in these conclusions. First, it will obviously be necessary to observe a larger sample of (ultra-) luminous infrared galaxies before one can be sure that the conclusions reached by Lutz et al. are generally applicable and before it is clear how our findings fit into an evolutionary scheme; this is the purpose of guaranteed time observations we will be carrying out with $I S O$ in the next year. Second, while the general conclusion that the luminous infrared galaxies discussed here are powered by massive stars is fairly robust, for several reasons the detailed constraints on the starburst properties are still fairly uncertain. Stellar-atmosphere models by Sellmaier et al. (1996) predict more energy output in the $20-50 \mathrm{eV}$ range than the Kurucz (1992) models used by Lutz et al. and thus require less-massive stars for the 


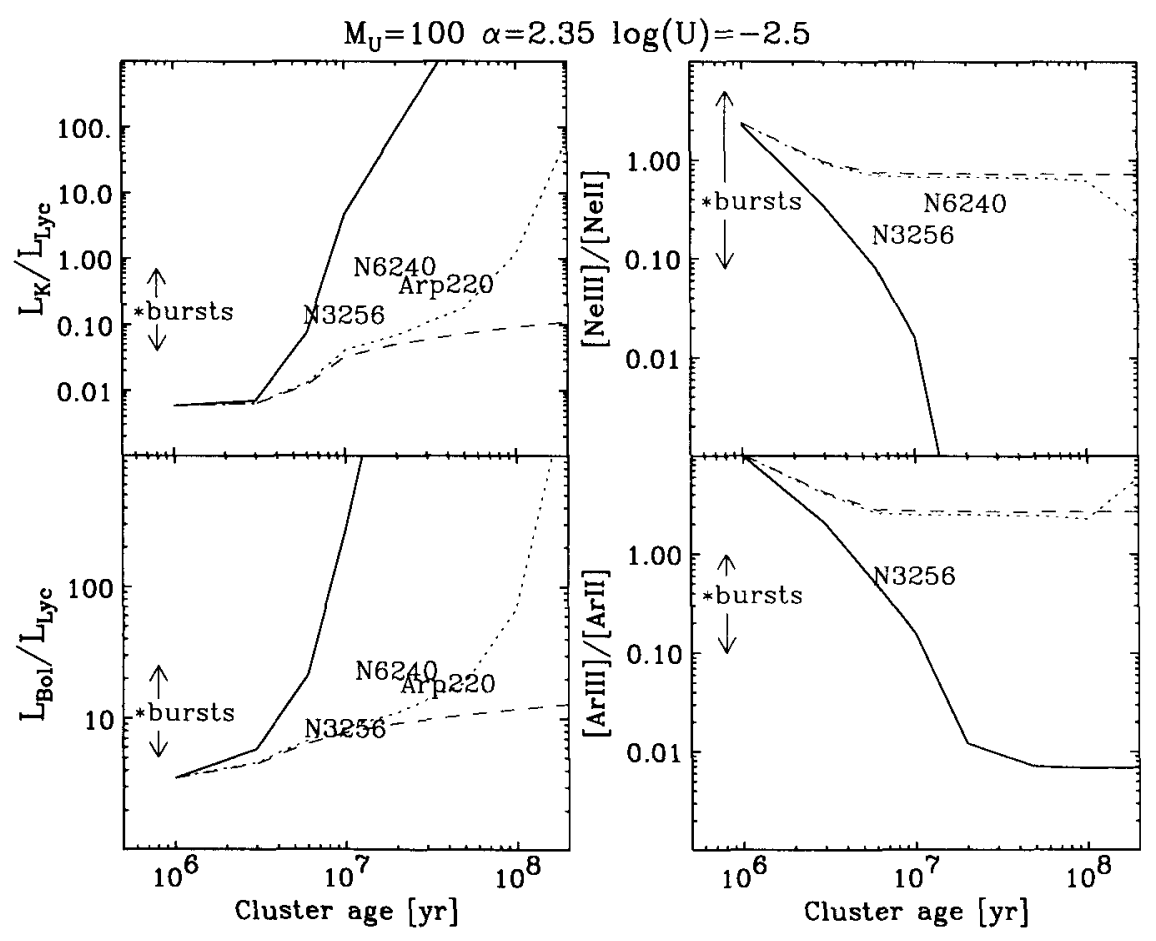

Figure 3. $\quad L_{B o l} / L_{L y c}, L_{K} / L_{L y c}$ and characteristic infrared line ratios for evolving star-cluster models (from Lutz et al. 1996). Lutz et al. used the Kovo \& Sternberg (1996) cluster code with Geneva tracks to calculate the numbers of stars in different parts of the HR diagram as a function of time and different star formation histories. In all models, we used a Salpeter IMF $(\gamma=-2.4)$, upper-mass cutoff of $100 M_{\odot}$ and lower-mass cutoff of $1 M_{\odot}$. Solid curves denote $\delta$-bursts (starformation rate $\propto \exp (-t / \Delta t)$ with $\Delta t=10^{6}$ years). Dotted curves denote extended bursts with $\Delta t=2 \times 10^{7}$ years, dashed curves are constant star-formation models. Line ratios were then calculated with the ION photoionization code (Netzer 1993) with an input spectrum obtained from coadding the appropriate Kurucz models for the cluster. In all cases, solar abundances were used in the stars and in the nebulae. 
same $[\mathrm{NeIII}] /[\mathrm{NeII}]$, etc., ratios. Line fluxes, line ratios, and extinction corrections all are subject to $\pm 30 \%$ calibration uncertainties and to uncertainties in the extinction curve. Finally, there are the issues of metallicity and the effects of dust within the $\mathrm{H}$ II regions which need to be addressed by more detailed future studies with $I S O$.

\section{The Central 100 Parsecs of the Seyfert 2 Galaxy NGC 1068}

At a distance of about $14 \mathrm{Mpc}\left(1^{\prime \prime}=68 \mathrm{pc}\right), \mathrm{NGC} 1068$ is one of the nearest and brightest Seyfert 2 galaxies. As its polarized optical spectrum shows the signature of a Seyfert 1 nucleus (Antonucci \& Miller 1985), it is generally considered as one of the prototypical cases for Seyfert unification models (Antonucci 1993).

Millimeter interferometry of molecular lines and sub-arcsecond imaging of the near-infrared continuum and several emission lines have given valuable new information on the distribution and dynamics of dense neutral gas and dust in the nucleus and central narrow-line region (NLR) and the relationship between NLR and the larger-scale disk of NGC 1068. Figure 4 shows $\sim 2^{\prime \prime}-4^{\prime \prime}$ resolution maps of ${ }^{12} \mathrm{CO} 1-0, \mathrm{CS} 2-1$ and HCN 1-0 emission (Tacconi et al. 1994, 1996, see also Planesas et al. 1991, Kaneko et al. 1992, Jackson et al. 1993, Helfer \& Blitz 1995, Papadopoulos et al. 1996). The CO line-emitting gas shows several components. First there is a tightly wound, twin spiral-arm structure associated with the starburst 'ring' at radius $\sim 13^{\prime \prime}(900 \mathrm{pc})$. Second, starting at the tips of the two spiral arms and following inward the near-infrared bar at position angle $\sim 35^{\circ}$ east of north is a gaseous bar or lane. At the center of this bar structure is a central concentration of molecular gas associated with the nucleus and the NLR on scales of a few hundred parsecs. The comparison of the CO maps with those of the high-density tracers HCN and CS immediately shows that the physical conditions of the circumnuclear gas are quite different from those of the gas in the ring/spiral arms. Tacconi et al. (1994), Sternberg et al. (1994), and Helfer \& Blitz (1995) derive $\mathrm{H}_{2}$ volume densities there of $10^{4}$ $10^{6} \mathrm{~cm}^{-3}$, column densities of about $5 \times 10^{22} \mathrm{H}_{2} \mathrm{~cm}^{-2}$, and gas temperatures $\geq 50 \mathrm{~K}$. While the gas kinematics in the spiral arms is dominated by galactic rotation, the gas associated with the bar appears to be streaming radially. The most likely interpretation is that the nuclear stellar bar causes gas to stream from the $\sim 1 \mathrm{kpc}$ scale to the circumnuclear environment. The current mass influx rate into the central few hundred parsecs may be a few $M_{\odot} \mathrm{yr}^{-1}$. Figure 5 shows an overlay of a $1^{\prime \prime} .5$ resolution CO $2-1$ map (Tacconi et al. 1996) on a $\sim 0^{\prime \prime} .8$ resolution map of the $2.12 \mu \mathrm{m} v=1-0 \mathrm{~S}(1)$ line of $\mathrm{H}_{2}$ (Thatte et al. 1996b). $\mathrm{H}_{2} \mathrm{~S}(1)$ and $\mathrm{CO} 2-1$ appear to be tracing the same gas. The higherresolution $\mathrm{H}_{2} \mathrm{~S}(1)$ map indicates that the circumnuclear gas has an oval or ring-like morphology (radius $\sim 1^{\prime \prime} .2$ or $80 \mathrm{pc}$ ), approximately centered on the near-infrared nucleus (cross in Fig. 5), but with a hot spot east-northeast of the nucleus. The $\mathrm{H}_{2} \mathrm{~S}(1)$ hot spot may also be present in the lower-resolution CO 2-1 map, indicating that there is a local concentration of molecular gas close to the peak of the NLR (which is centered NE of the nucleus as well). The kinematics of the dense circumnuclear gas are also dominated by streaming, with projected peak velocities of about $\pm 100 \mathrm{~km} \mathrm{~s}^{-1}$ (Fig. 5, Tacconi et al. 1996). The circumnuclear ring may represent gas in resonance orbits that is orbiting in the 


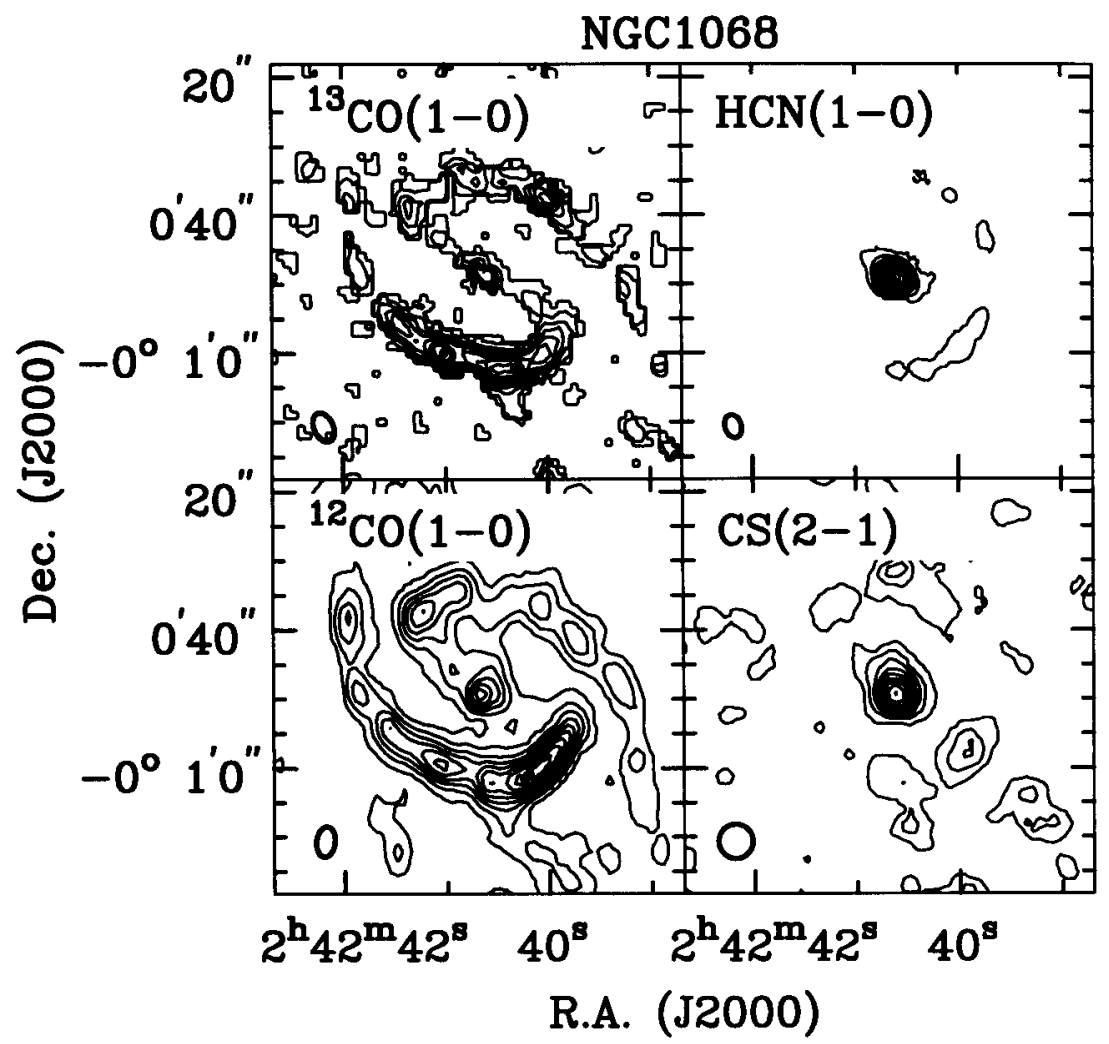

Figure 4. ${ }^{12} \mathrm{CO} 1-0$ (left, $4^{\prime \prime}$ resolution) and $\mathrm{HCN} 1-0$ (right, $2^{\prime \prime} .5$ resolution) mm-interferometer maps of NGC 1068, taken with the Plateau de Bure mm-interferometer (Tacconi et al. 1994, 1996). 

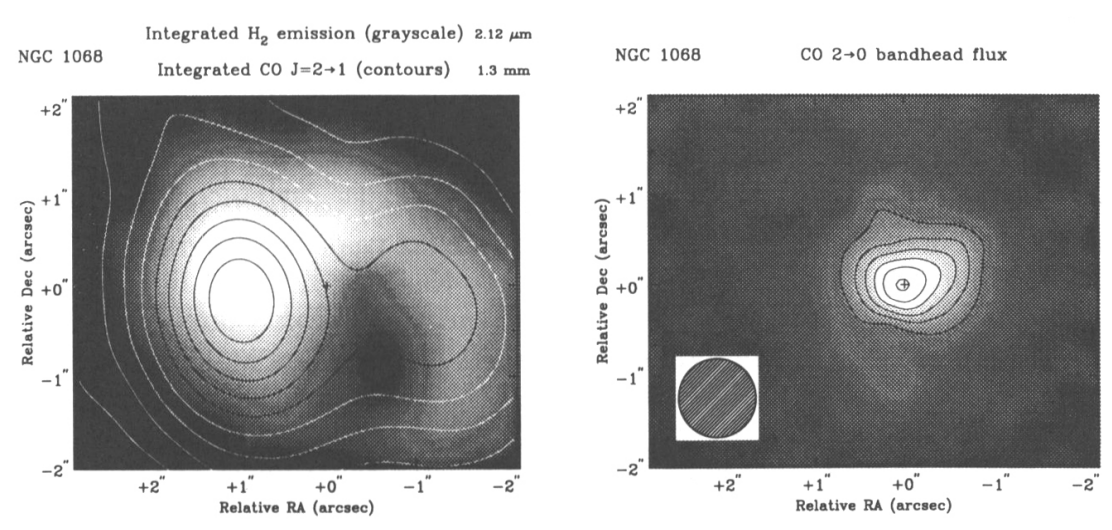

Figure 5. Left: Overlay of a $1^{\prime \prime} .5$ resolution ${ }^{12} \mathrm{CO} 2-1$ map (contours, Tacconi et al. 1996) on a $0^{\prime \prime} .83 \mathrm{D}$ map of the $2.12 \mu \mathrm{m} \mathrm{H} \mathrm{H}_{2}(v=1-0) \mathrm{S}(1)$ (grey-scale, Thatte et al. 1996b), showing the circumnuclear environment of NGC 1068. Right: 3D map of the $2.3 \mu \mathrm{m} v=0-2 \mathrm{CO}$ bandhead absorption flux, tracing the distribution of red giant/supergiant stars. The cross in both panels denotes the position of the compact near-infrared hot dust source which likely marks the location of the AGN.

central bar potential and interacting with radiation and winds/jets from the nuclear source. The intense NLR emission northeast of the nucleus may then mark exactly this interaction zone.

There is another concentration of interstellar dust (and gas) on yet smaller scales. The near-infrared spectrum longward of about $2.2 \mu \mathrm{m}$ is dominated by a steeply rising red continuum, probably caused by hot $(T \approx 700 \mathrm{~K})$ dust grains in the immediate vicinity of the nuclear source. Recent speckle observations with pupil masks on the 3.5-m NTT show that the diameter of the dust source at $2.3 \mu \mathrm{m}$ is about 30 to 50 milliarcseconds (2-3.5 pc, Thatte et al. 1996a). The minimum hydrogen column density of the compact dust source, assuming unit filling factor, optically thin emission, and a gas-to-dust mass ratio of 100 , is a few times $10^{21} \mathrm{~cm}^{-2}$ ( $A_{V}$ greater than a few magnitudes). The real column densities could be much greater, however, and this central dust concentration may be identical with the postulated parsec-scale torus (Krolik \& Begelman 1986). The millimeter and near-infrared observations thus show very clearly that interstellar material is fed into the nucleus via a hierarchy of scales/mechanisms. The stellar bar(s) play a key role in this transport mechanism, in agreement with theoretical models (e.g., Shlosman et al. 1990).

Another important issue is the importance of stars in the energetics of the nucleus of NGC 1068. While stellar light dominates the optical continuum in the central few arcseconds (e.g., Koski 1978), its luminosity and spatial distribution are not very well known. Figure 5 shows recent $3 \mathrm{D}$ imaging observations of the $2.3 \mu \mathrm{m} \mathrm{CO}$ band-head absorption flux and equivalent width (Thatte et al. 1996a). The CO absorption traces late-type giants and supergiants. The stellar- 
absorption equivalent width has a minimum toward the nucleus (due to dilution by the nuclear hot-dust emission discussed above), but the flux map in Fig. 5 indicates the presence of a fairly well-defined, compact (intrinsic diameter $\sim 0^{\prime \prime} .7$ or $50 \mathrm{pc}$ ) nuclear stellar cluster. Correcting the dereddened $H$ and $K$ band spectroscopic data for the dust emission and comparing the resulting nearinfrared stellar luminosity $\left(L(1.9-2.4 \mu \mathrm{m})=2 \times 10^{8} L_{\odot}\right)$ to the total (stellar + gas + nuclear) dynamical mass derived from the stellar velocity dispersion, Oliva et al. (1995) and Thatte et al. (1996a) find that the near-infrared light comes from stars that have formed less than a few hundred million years ago. This upper limit applies if the entire dynamical mass is due to stars and if the entire nuclear cluster formed at that time. In this case the bolometric luminosity of the nuclear stellar cluster is about $10-20 \%$ of the nuclear luminosity $\left(1.5 \times 10^{11} L_{\odot}\right)$. If the cluster is younger (because an old stellar cluster contributes to the mass, but little to the near-infrared light) the nuclear stellar luminosity may be larger. These observations give a first hint that stars as well as a compact active nucleus contribute significantly to the nuclear luminosity of NGC 1068, as proposed by Terlevich (1995).

Acknowledgments. We thank the members of the SRON-MPE SWS team (especially O. Bauer, D. Beintema, E. Egami, T. deGraauw, R. Katterloher, L. Haser, D. Kunze, D. Rigopoulou, and E. Wieprecht) and the SWS IDT for their hard and dedicated work which has made these observations possible. We are also grateful to T. Alexander, A. Moorwood, O. Kovo, and H. Netzer for theoretical input. J. Gallimore, A. Krabbe, H. Kroker, and R. Maiolino helped with the 3D observations.

\section{References}

Antonucci, R. R. J., \& Miller, J. S. 1985, ApJ, 297, 621.

Antonucci, R. R. J. 1993, ARAA, 31, 473.

Armus, L., Neugebauer, G., Soifer, B. T. \& Matthews, K. 1995, AJ, 110, 2610.

Armus, L., Shupe, D. L., Matthews, K., Soifer, B. T., \& Neugebaner, G. 1996, ApJ, in press.

Bryant, P. M., \& Scoville, N.Z. 1996, ApJ, 457, 678.

Cid Fernandes, R., Jr., \& Terlevich, R. 1995, MNRAS, 272, 423.

Condon, J. J., \& Yin, Q. F. 1990, ApJ, 357, 97.

Condon, J. J., Helou, G., Sanders, D. B., \& Soifer, B. T. 1990, ApJS, 73, 359.

DeGraauw, Th., et al. 1996, A\&A, in press.

Downes, D., Solomon, P. M., \& Radford, S. J. E. 1995, ApJ, 453, L65.

Doyon, R., Puxley, P. J., \& Joseph, R. D. 1992, ApJ, 397, 117.

Fischer, J., et al. 1996, A\&A, in press.

Foerster, N., et al. 1996, in prep.

Genzel, R., et al. 1995, ApJ, 444, 129.

Goldader, J.D., Joseph, R. D., Doyon, R., \& Sanders, D. B. 1995, ApJ, 444, 97.

Goldader, J. D., Joseph, R. D., Doyon, R., \& Sanders, D. B. 1996, in press.

Helfer, T., \& Blitz, L. 1995, ApJ, 450, 90. 
Jackson, J. M., Paglione, T.A.D., Ishizuki, S., \& Rieu, N.-Q. 1993, ApJ, 418, L13.

Kaneko, N., et al. 1992, PASJ, 44, 341.

Heckman, T. M., Armus, L., \& Miley, G. K. 1990, ApJS, 74, 833.

Kim, D.C., Sanders, D. B., Veilleux, S., Mazzarella, J. M., \& Soifer, B. T. 1995, ApJS, 98, 129.

Kotilainen, J.K., Forbes, D.A., Moorwood, A.F.M., van der Werf, P.P., \& Ward, M. 1996, A\&A, in press.

Koski, A. T. 1978, ApJ, 223, 56.

Kovo, O., \& Sternberg, A. 1996, in prep.

Krabbe, A., Stermberg, A., \& Genzel, R. 1994, ApJ, 425, 72.

Krabbe, A., et al. 1995, ApJ, 447, L95.

Krolik, J. H., \& Begelman, M. C. 1986, ApJ, 308, L55.

Kunze, D., et al. 1996, A\&A, in press.

Kurucz, R. L. 1992, Rev. Mex. Astron. Astrofis., 23, 181.

Larkin, J.E., Armus, L., Knop, R. A., Matthews, K., \& Soifer, B. T. 1995, ApJ, 452,599 .

Lester, D., Harvey, P. M., \& Carr, J. 1988, ApJ, 329, 641.

Lonsdale, C. J., Smith, H.E., \& Lonsdale, C. J. 1993, ApJ, 327, L41.

Lutz, D., et al. 1996a, A\&A, in press.

Meynet, G., Maeder, A., Schaller, G., Schaerer, D., \& Charbonnel, C. 1994, A\&A, 103, 97.

Moorwood, A. F. M. 1994, ApJ, 429, 602.

Moorwood, A.F.M., et al. 1996, A\&A, in press.

Netzer, H. 1993, ApJ. 411, 594.

Oliva, E., Salvati, M., Moorwood, A. F. M., \& Marconi, A. 1994, A\&A, 288, 457.

Oliva, E. ,Origlia, L., Kotilainen, J. K., \& Moorwood, A. F. M. 1995, A\&A, 301, 55 .

Papadopoulos, P.P., Seaquist, E. R., \& Scoville, N.Z. 1996, ApJ, 465, 173.

Planesas, P., Scoville, N.Z., \& Myers, S. T. 1991, ApJ, 369, 364.

Rieke, G.H., Cutri, R., Black, J.H., Kailey, W.F., McAlary, C.W., Lebofsky, M.J. \& Elston, R. 1985, ApJ, 290, 116.

Rieke, G. H., Loken, K., Rieke, M. J., \& Tamblyn, P. 1993, ApJ, 412, 99.

Rigopoulou, D., et al. 1996, A\&A, in press.

Rigopoulou, D., Lawrence, A., White, G.J., Rowan-Robinson, M., \& Church, S. E. 1996, A\&A, 305, 747.

Rowan-Robinson, M., \& Efstathiou, A. 1993, MNRAS, 263, 675.

Sanders, D. B., \& Mirabel, I. F. 1985, ApJ, 298, L31.

Sanders, D. B., \& Mirabel, I. F. 1996, ARAA, 34, in press.

Sanders, D. B., et al. 1988, ApJ, 325, 74.

Satyapal, S., et al. 1995, ApJ, 448, 611. 
Scoville, N.Z., Yun, M., Brown, R.L., \& VandenBout, P.A. 1995, ApJ, 449, L109.

Sellmaier, F., Yamamoto, T., Pauldrach, A. W. A., \& Rubin, R. H. 1996, A\&A, $305, \mathrm{~L} 37$.

Shlosman, I., Begelman, M. C., \& Frank, J. 1990, Nature, 345, 679.

Soifer, B. T, Houck, J. R., \& Neugebauer, G. 1987, ARAA, 25, 187.

Sternberg, A., Genzel, R., \& Tacconi, L. J. 1994, ApJ, 436, L131.

Sturm, E., et al. 1996, A\&A, in press.

Tacconi, L. J., Genzel, R., Blietz, M., Cameron, M., Harris, A. I., \& Madden, S. 1994, ApJ, 426, L77.

Tacconi, L. J., et al. 1996, in prep.

Tacconi-Garman, L. E., Sternberg, A., \& Eckart, A. 1996, ApJ, in press.

Thatte, N., Quirrenbach, A., et al. 1996a, in prep.

Thatte, N., Tacconi, L. J., et al. 1996b, in prep.

Thronson, H. A., Majewski, S., Descartes, L., \& Hereld, M. 1990, ApJ, 364, 456. van der Werf, P.P., et al. 1993, ApJ, 405, 522.

Veilleux, S., Kim, D. C., Sanders, D. B., Mazarella, J.M., \& Soifer, B. T. 1995, ApJS, 98, 171.

Vigroux, L., et al. 1996, A\&A, in press.

Wang, Z., Scoville, N.Z., \& Sanders, D. B. 1991, ApJ. 386, 112.

Yun, M.S., \& Scoville, N.Z. 1995, ApJ, 451, L45. 